\title{
Towards optimized Schwarz methods for the Navier-Stokes equations
}

\author{
Eric Blayo • David Cherel • Antoine \\ Rousseau
}

Received: date / Accepted: date

\begin{abstract}
This paper presents a study of optimized Schwarz domain decomposition methods for Navier-Stokes equations. Once discretized in time, optimal transparent boundary conditions are derived for the resulting Stokes equations, and a series of local approximations for these nonlocal conditions are proposed. Their convergence properties are studied, and numerical simulations are conducted on the test case of the driven cavity with two subdomains. It is shown that conditions involving one or two degrees of freedom can improve the convergence properties of the original algorithm.
\end{abstract}

Keywords Domain decomposition · Optimized boundary conditions · Navier Stokes equations · Schwarz Alternating Method

PACS $65 \mathrm{~N} 55 \cdot 76 \mathrm{D} 05$

\section{Introduction}

A number of domain decomposition approaches have already been proposed for the numerical resolution of Navier-Stokes equations on parallel computers. These approaches can be barely classified into a few main families: algebraic methods working on the discrete matrix system (e.g. $[15,23,1])$, variational methods minimizing a misfit at the interfaces between subdomains (e.g. $[8,14])$, and Schwarz alternating methods. This last class of methods has the advantage of being quite non intrusive, since it does not require modifications in the original computation code except for the boundary conditions at the interface between subdomains. Moreover the Schwarz formulation is a natural framework for coupling different models, which makes it particularly relevant for a very large number of applications. This aspect is our main motivation for the present work, which can be seen as a step in the direction of coupling different models in fluid dynamics.

E. Blayo $^{1,2,3}$, D. Cherel ${ }^{1,2,3}$, A. Rousseau ${ }^{3}$

1 Univ. Grenoble Alpes, LJK, F-38000 Grenoble, France

2 CNRS, LJK, F-38000 Grenoble, France

3 Inria

E-mail: Eric.Blayo@imag.fr 
A number of previous works deal with Schwarz-type algorithms for Stokes, NavierStokes and Oseen (i.e. linearized Navier-Stokes) systems. They study either DirichletDirichlet [26], Dirichlet-Neumann [29], Neumann-Neumann [22], or Robin-Robin $[21,19]$ algorithms. See also [27]. In this paper, our objective is to derive more general optimized conditions, leading to an efficient convergence. To achieve this, we will follow the well-known strategy (e.g. $[12,7])$ which consists first in deriving exact transparent operators (which are pseudo-differential, hence unusable for practical applications) and second in approximating them by differential operators. Note that, using a different approach based on Smith factorization, [3] have also proposed an efficient domain decomposition algorithm for Stokes and Oseen systems.

The non dimensional Navier-Stokes system in a domain $\Omega$ with boundary $\partial \Omega$ reads:

$$
\left\{\begin{array}{l}
\frac{\partial \boldsymbol{v}}{\partial t}+\boldsymbol{v} \nabla \boldsymbol{v}-\frac{1}{\operatorname{Re}} \Delta \boldsymbol{v}+\nabla p=0 \text { in } \Omega \\
\operatorname{div} \boldsymbol{v}=0 \text { in } \Omega \\
\boldsymbol{v}=\boldsymbol{g} \text { on } \partial \Omega
\end{array}\right.
$$

where Re is the Reynolds number. In the present work, we will not consider waveform relaxation (or, in other words, global-in-time) domain decomposition methods, but will start with a time discretization of the Navier-Stokes system:

$$
\left\{\begin{array}{l}
\frac{\boldsymbol{v}^{n+1}-\boldsymbol{v}^{n}}{\Delta t}+\boldsymbol{v}^{n} \nabla \boldsymbol{v}^{n}-\frac{1}{\operatorname{Re}} \Delta \boldsymbol{v}^{n+1}+\nabla p^{n+1}=0 \text { in } \Omega \\
\operatorname{div} \boldsymbol{v}^{n+1}=0 \text { in } \Omega \\
\boldsymbol{v}^{n+1}=\boldsymbol{g}^{n+1} \text { on } \partial \Omega .
\end{array}\right.
$$

Then one has to solve at each time step a Stokes problem:

$$
\left\{\begin{array}{l}
\frac{1}{\Delta t} \boldsymbol{v}-\frac{1}{\operatorname{Re}} \Delta \boldsymbol{v}+\nabla p=\boldsymbol{f} \text { in } \Omega \\
\operatorname{div} \boldsymbol{v}=0 \text { in } \Omega \\
\boldsymbol{v}=\boldsymbol{g} \text { on } \partial \Omega
\end{array}\right.
$$

For the sake of simplicity, we will consider the 2-D $x-z$ case in this work.

This paper is structured as follows. In Section 2, we will recall the framework of Schwarz algorithms for domain decomposition, and derive transparent boundary conditions following the methodology described in the seminal papers $[5,10,9]$. A series of approximations for these transparent conditions will be proposed in Section 3, and their convergence properties will be studied. Then some numerical simulations on the well known test case of the driven cavity decomposed into two subdomains will be described in Section 4.

\section{Schwarz algorithm and theoretical aspects}

In this section, we set the domain decomposition method (DDM) framework of this study, namely the iterative Schwarz algorithm (see $[24,16,17]$ ). We introduce the analytical expression of the errors (evolving with the iterative process), and provide a generic expression for the convergence rate. We draw the reader's attention to the fact that no specific interface condition is introduced in this section (the operators $S_{j}$ below remain undefined). Naturally, these conditions are required for the actual implementation of the algorithm, and will be fully determined in Section 3. 
2.1 DDM framework and equations for the errors

The computational domain $\Omega$ is split in two subdomains: $\Omega=\Omega_{1} \cup \Omega_{2}$, with possible overlapping. Their internal boundaries, denoted $\Gamma_{1}$ and $\Gamma_{2}$, are defined by

$$
\Gamma_{j}=\partial \Omega_{j} \cap \bar{\Omega}_{j^{\prime}} \quad j=1,2 \quad \text { where } j^{\prime}=3-j
$$

In the particular case of non overlapping subdomains, $\Gamma_{1}=\Gamma_{2}=\Gamma$. On each of the two subdomains $\Omega_{j}$, we look for the solution of the following system of equations:

$$
\left\{\begin{array}{l}
\frac{1}{\Delta t} \boldsymbol{v}_{j}^{m}-\frac{1}{\operatorname{Re}} \Delta \boldsymbol{v}_{j}^{m}+\nabla p_{j}^{m}=\boldsymbol{f} \text { in } \Omega_{j} \\
\operatorname{div} \boldsymbol{v}_{j}^{m}=0 \text { in } \Omega_{j} \\
\boldsymbol{v}_{j}^{m}=\boldsymbol{g} \text { on } \partial \Omega_{j} \backslash \Gamma_{j} \\
\mathrm{IBC} \text { on } \Gamma_{j},
\end{array}\right.
$$

where $I B C$ stands for interface boundary conditions. Although we postpone the complete definition of these conditions to Section 3, we will use the generic conditions of the form

$$
-\boldsymbol{\sigma}_{j}^{m} \cdot \boldsymbol{n}_{j}-S_{j} \boldsymbol{v}_{j}^{m}=-\boldsymbol{\sigma}_{j^{\prime}}^{m-1} \cdot \boldsymbol{n}_{j}-S_{j} \boldsymbol{v}_{j^{\prime}}^{m-1} \text { on } \Gamma_{j},
$$

where $\boldsymbol{\sigma}_{j}^{m}=1 / \operatorname{Re} \nabla \boldsymbol{v}_{j}^{m}-p_{j}^{m}$ Id is the stress tensor ${ }^{1}$ in the domain $\Omega_{j}, \boldsymbol{n}_{j}$ is the outward normal vector and $S_{j}$ is a generic operator, to be defined later on. These conditions, thanks to the convergence properties analyzed in Section 3.2 below, ensure that both velocities and fluxes are continuous through the interfaces $\Gamma_{1}$ and $\Gamma_{2}$ at the end of the iterative process.

In the system of equations (4), the integer $m$ denotes the iteration index in the Schwarz algorithm. Since the right-hand side $f$ does not depend on $m$, the DDM error is solution of the following system:

$$
\left\{\begin{array}{l}
\frac{1}{\Delta t} \boldsymbol{e}_{j}^{m}-\frac{1}{\operatorname{Re}} \Delta \boldsymbol{e}_{j}^{m}+\nabla q_{j}^{m}=0 \text { in } \Omega_{j} \\
\operatorname{div} \boldsymbol{e}_{j}^{m}=0 \text { in } \Omega_{j} \\
\boldsymbol{e}_{j}^{\boldsymbol{m}}=0 \text { on } \partial \Omega_{j} \backslash \Gamma_{j} \\
-\boldsymbol{\sigma}_{j}^{m} \cdot \boldsymbol{n}_{j}-S_{j} \boldsymbol{e}_{j}^{m}=-\boldsymbol{\sigma}_{j^{\prime}}^{m-1} \cdot \boldsymbol{n}_{j}-S_{j} \boldsymbol{e}_{j^{\prime}}^{m-1} \text { on } \Gamma_{j},
\end{array}\right.
$$

where $\boldsymbol{e}_{j}=\left(e_{j}^{u}, e_{j}^{w}\right)=\boldsymbol{v}_{\mid \Omega_{j}}-\boldsymbol{v}_{j}$ and $q_{j}=p_{\mid \Omega_{j}}-p_{j}$ denote the velocity and pressure errors in $\Omega_{j}$, and where $\boldsymbol{\sigma}_{j}^{m}$ deals now with the error fields.

\subsection{Analytical expression of the error in infinite domains}

In order to compute approximate solutions of equations (6), we assume that both domains $\left(\Omega_{j}\right)_{j=1,2}$ are semi-infinite in $x$, and infinite in $z: \Omega_{1}=(-\infty, L) \times \mathbb{R}$ and

\footnotetext{
1 We consider here the non symmetric form of the stress tensor. The alternative consisting in using its symmetric form will be discussed in Section 5 .
} 
$\Omega_{2}=(0,+\infty) \times \mathbb{R}$, with $L \geq 0$. The system of equations (6) becomes

$$
\left\{\begin{array}{l}
\frac{1}{\Delta t} \boldsymbol{e}_{j}^{m}-\frac{1}{\operatorname{Re}} \Delta \boldsymbol{e}_{j}^{m}+\nabla q_{j}^{m}=0 \text { in } \Omega_{j} \\
\operatorname{div} \boldsymbol{e}_{j}^{m}=0 \text { in } \Omega_{j} \\
\boldsymbol{e}_{\boldsymbol{j}}^{\boldsymbol{m}} \rightarrow 0 \text { as }(x, z) \rightarrow \pm \infty \\
-\boldsymbol{\sigma}_{j}^{m} \cdot \boldsymbol{n}_{j}-S_{j} \boldsymbol{e}_{j}^{m}=-\boldsymbol{\sigma}_{j^{\prime}}^{m-1} \cdot \boldsymbol{n}_{j}-S_{j} \boldsymbol{e}_{j^{\prime}}^{m-1} \text { on } \Gamma_{j},
\end{array}\right.
$$

with $\Gamma_{1}=\{L\} \times \mathbb{R}$ and $\Gamma_{2}=\{0\} \times \mathbb{R}$.

We now proceed to a Fourier transform in the vertical direction, denoting by $k$ the frequency variable associated to the space variable $z$, and $\hat{f}$ the Fourier transform of any function $f$. The system of equations (7) becomes:

$$
\left\{\begin{array}{l}
\frac{1}{\Delta t} \hat{\boldsymbol{e}}_{j}^{m}-\frac{1}{\operatorname{Re}}\left(\frac{d^{2} \hat{\boldsymbol{e}}_{j}^{m}}{d x^{2}}-k^{2} \hat{\boldsymbol{e}}_{j}^{m}\right)+\left(\begin{array}{c}
\frac{d \hat{q}_{j}^{m}}{d x} \\
i k \hat{q}_{j}^{m}
\end{array}\right)=\mathbf{0} \text { in } \Omega_{j} \\
\frac{d \hat{e}_{j}^{u, m}}{d x}+i k \hat{e}_{j}^{w, m}=0 \text { in } \Omega_{j} \\
\hat{\boldsymbol{e}}_{j}^{m} \rightarrow \mathbf{0} \text { when }|x| \rightarrow \infty \\
-\hat{\boldsymbol{\sigma}}_{j}^{m} \cdot \boldsymbol{n}_{j}-\hat{S}_{j} \hat{\boldsymbol{e}}_{j}^{m}=-\hat{\boldsymbol{\sigma}}_{j^{\prime}}^{m-1} \cdot \boldsymbol{n}_{j}-\hat{S}_{j} \hat{\boldsymbol{e}}_{j^{\prime}}^{m-1} \text { on } \Gamma_{j} .
\end{array}\right.
$$

As in [9] and [18], we look for solutions of this system under the generic form:

$$
E_{j}^{m}:=\left(\hat{\boldsymbol{e}}_{j}^{m}, \hat{q}_{j}^{m}\right)^{T}(x)=\boldsymbol{\Phi}_{j}^{m} e^{\xi x} .
$$

Introducing this expression in (8), we have the following system:

$$
\left(\begin{array}{ccc}
\frac{1}{\Delta t}+\frac{k^{2}-\xi^{2}}{\operatorname{Re}} & 0 & \xi \\
0 & \frac{1}{\Delta t}+\frac{k^{2}-\xi^{2}}{\operatorname{Re}} i k \\
\xi & i k & 0
\end{array}\right) \boldsymbol{\Phi}_{j}^{m}=0 .
$$

A necessary and sufficient condition to obtain a non-zero solution to system (9) is to have a singular matrix, which leads to four possible values for $\xi$ :

$$
\xi_{1}=\lambda, \xi_{2}=|k|, \xi_{3}=-\lambda \text { and } \xi_{4}=-|k|,
$$

where

$$
\lambda=\sqrt{\frac{\operatorname{Re}}{\Delta t}+k^{2}} .
$$

The solutions of (9) are thus linear combinations of four modes

$$
E_{j}^{m}=\sum_{l=1}^{4} \gamma_{j, l}^{m} \Phi_{l} e^{\xi_{l} x}
$$

where $\left(\boldsymbol{\Phi}_{\boldsymbol{l}}\right)_{1 \leq l \leq 4}$ are the eigenvectors (corresponding to eigenvalue 0 ) associated with each of the $\xi_{l}$ :

$$
\boldsymbol{\Phi}_{\mathbf{1}}=\left(\begin{array}{c}
i k \\
-\lambda \\
0
\end{array}\right), \boldsymbol{\Phi}_{\mathbf{2}}=\left(\begin{array}{c}
-|k| \\
-i k \\
1 / \Delta t
\end{array}\right), \boldsymbol{\Phi}_{\mathbf{3}}=\left(\begin{array}{c}
i k \\
\lambda \\
0
\end{array}\right) \text { and } \boldsymbol{\Phi}_{\mathbf{4}}=\left(\begin{array}{c}
|k| \\
-i k \\
1 / \Delta t
\end{array}\right) \text {. }
$$


Thanks to the condition (8c), since $\xi_{1}, \xi_{2}>0$ and $\xi_{3}, \xi_{4}<0$, only two modes are retained in the expression of $E_{j}^{m}$ in each of the subdomains. We have:

$$
E_{1}^{m}(x)=\sum_{l=1}^{2} \gamma_{1, l}^{m} \boldsymbol{\Phi}_{l} e^{\xi_{l} x} \quad \text { and } \quad E_{2}^{m}(x)=\sum_{l=3}^{4} \gamma_{2, l}^{m} \boldsymbol{\Phi}_{l} e^{\xi_{l} x}
$$

Equipped with these expressions for the errors $E_{j}^{m}$, we now want to make use of interface conditions $(8 \mathrm{~d})$ to study the corresponding convergence rates.

\subsection{Theoretical convergence rates}

We first simplify the expressions of errors by expressing the pressure error term thanks to the vertical component of equation (8a)

$$
\hat{q}_{j}^{m}=\frac{i}{k}\left[\left(\frac{1}{\Delta t}+\frac{k^{2}}{\operatorname{Re}}\right) \hat{\boldsymbol{e}}_{j}^{m}-\frac{1}{\operatorname{Re}} \frac{d^{2} \hat{\boldsymbol{e}}_{j}^{m}}{d x^{2}}\right] \cdot(0,1)^{t}
$$

The interface boundary conditions $(8 \mathrm{~d})$ on $\Gamma_{1}(x=L)$ and $\Gamma_{2}(x=0)$ now reads:

$$
\begin{gathered}
M \frac{d^{2} \hat{\boldsymbol{e}}_{1}^{m}}{d x^{2}}+N \frac{d \hat{\boldsymbol{e}}_{1}^{m}}{d x}+P \hat{\boldsymbol{e}}_{1}^{m}-\hat{S}_{1} \hat{\boldsymbol{e}}_{1}^{m}=M \frac{d^{2} \hat{\boldsymbol{e}}_{2}^{m-1}}{d x^{2}}+N \frac{d \hat{\boldsymbol{e}}_{2}^{m-1}}{d x}+P \hat{\boldsymbol{e}}_{2}^{m-1}-\hat{S}_{1} \hat{\boldsymbol{e}}_{2}^{m-1} \\
-M \frac{d^{2} \hat{\boldsymbol{e}}_{2}^{m}}{d x^{2}}-N \frac{d \hat{\boldsymbol{e}}_{2}^{m}}{d x}-P \hat{\boldsymbol{e}}_{2}^{m}-\hat{S}_{2} \hat{\boldsymbol{e}}_{2}^{m}=-M \frac{d^{2} \hat{\boldsymbol{e}}_{1}^{m-1}}{d x^{2}}-N \frac{d \hat{\boldsymbol{e}}_{1}^{m-1}}{d x}-P \hat{\boldsymbol{e}}_{1}^{m-1}-\hat{S}_{2} \hat{\boldsymbol{e}}_{1}^{m-1},
\end{gathered}
$$

with the matrices $M, N$ and $P$ defined as follows

$$
M=-\frac{1}{\operatorname{Re}}\left(\begin{array}{cc}
0 & \frac{i}{k} \\
0 & 0
\end{array}\right), N=-\frac{1}{\operatorname{Re}} I_{2} \text { and } P=-\frac{1}{\operatorname{Re}}\left(\begin{array}{cc}
0 & -\frac{i}{k} \lambda^{2} \\
0 & 0
\end{array}\right) .
$$

Thanks to (11), we have

$$
\hat{\boldsymbol{e}}_{1}^{m}(x)=M_{12} e^{\xi_{12} x} \gamma_{12}^{m} \quad \text { and } \quad \hat{\boldsymbol{e}}_{2}^{m}(x)=M_{34} e^{\xi_{34} x} \gamma_{34}^{m}
$$

with

$$
\begin{aligned}
& M_{12}=\left(\begin{array}{c}
i k-|k| \\
-\lambda-i k
\end{array}\right), e^{\xi_{12} x}=\left(\begin{array}{cc}
e^{\xi_{1} x} & 0 \\
0 & e^{\xi_{2} x}
\end{array}\right) \text { and } \gamma_{12}^{m}=\left(\begin{array}{c}
\gamma_{1,1}^{m} \\
\gamma_{1,2}^{m}
\end{array}\right) \\
& M_{34}=\left(\begin{array}{cc}
i k & |k| \\
\lambda & -i k
\end{array}\right), e^{\xi_{34} x}=\left(\begin{array}{cc}
e^{\xi_{3} x} & 0 \\
0 & e^{\xi_{4} x}
\end{array}\right) \text { and } \gamma_{34}^{m}=\left(\begin{array}{c}
\gamma_{2,3}^{m} \\
\gamma_{2,4}^{m}
\end{array}\right)
\end{aligned}
$$


Then (12) and (13) can be rewritten as $\hat{H}_{11} \gamma_{12}^{m}=\hat{H}_{12} \gamma_{34}^{m-1}$ and $\hat{H}_{22} \gamma_{34}^{m}=$ $\hat{H}_{21} \gamma_{12}^{m-1}$ where

$$
\begin{aligned}
& \hat{H}_{11}=\left[M M_{12}\left(\begin{array}{cc}
\xi_{1}^{2} & 0 \\
0 & \xi_{2}^{2}
\end{array}\right)+N M_{12}\left(\begin{array}{cc}
\xi_{1} & 0 \\
0 & \xi_{2}
\end{array}\right)+P M_{12}-\hat{S}_{1} M_{12}\right] e^{\xi_{12} L} \\
& \hat{H}_{12}=\left[M M_{34}\left(\begin{array}{cc}
\xi_{3}^{2} & 0 \\
0 & \xi_{4}^{2}
\end{array}\right)+N M_{34}\left(\begin{array}{cc}
\xi_{3} & 0 \\
0 & \xi_{4}
\end{array}\right)+P M_{34}-\hat{S}_{1} M_{34}\right] e^{\xi_{34} L} \\
& \hat{H}_{22}=-M M_{34}\left(\begin{array}{cc}
\xi_{3}^{2} & 0 \\
0 & \xi_{4}^{2}
\end{array}\right)-N M_{34}\left(\begin{array}{cc}
\xi_{3} & 0 \\
0 & \xi_{4}
\end{array}\right)-P M_{34}-\hat{S}_{2} M_{34} \\
& \hat{H}_{21}=-M M_{12}\left(\begin{array}{cc}
\xi_{1}^{2} & 0 \\
0 & \xi_{2}^{2}
\end{array}\right)-N M_{12}\left(\begin{array}{cc}
\xi_{1} & 0 \\
0 & \xi_{2}
\end{array}\right)-P M_{12}-\hat{S}_{2} M_{12} .
\end{aligned}
$$

We thus obtain the following recursive property for the errors:

$$
\gamma_{12}^{m}=\hat{H}_{11}^{-1} \hat{H}_{12} \hat{H}_{22}^{-1} \hat{H}_{21} \gamma_{12}^{m-2}
$$

which means that the growth rate of the error is directly related to the spectral radius of the amplification matrix

$$
\hat{H}=\hat{H}_{11}^{-1} \hat{H}_{12} \hat{H}_{22}^{-1} \hat{H}_{21} \text {. }
$$

More precisely, the convergence rate of the Schwarz algorithm is equal to the square root of this radius in case of an additive version (as written in (12)-(13)-(15)), and to the radius itself in case of a multiplicative version (since index $m-1$ is then replaced by $m$ in the r.h.s. of (13), which means that $m-2$ is replaced by $m-1$ in (15)). In order to ensure the convergence of the Schwarz algorithm for all possible frequencies $k \in\left[k_{\min }, k_{\max }\right]$, we have then to choose appropriately the operator $S_{j}$ such that (e.g. $\left.[7,12,13]\right)$

$$
\rho(\hat{H})=\max _{k \in\left[k_{\min }, k_{\max }\right]} \max _{\mu \in S p(\hat{H})}|\mu(k)|<1 .
$$

The objective of Section 3 below is to specify the operator $S_{i}$ that should be used in numerical simulations in order to ensure the well-posedness of (8) and to cancel (or minimize) the spectral radius of the amplification matrix $\hat{H}$.

\section{Transparent boundary conditions and their approximations}

This section focuses on the interface boundary conditions that should be used in Equation (8d). More specifically, we will look for operators $S_{i}$ that will be both efficient in term of convergence rate, and numerically tractable.

\subsection{Exact transparent conditions}

It is easy to check that the right-hand sides of equations (12) and (13), thus of (8d), cancel for

$$
\begin{aligned}
& \hat{S}_{1}^{*}=M M_{34}\left(\begin{array}{cc}
\xi_{3}^{2} & 0 \\
0 & \xi_{4}^{2}
\end{array}\right) M_{34}^{-1}+N M_{34}\left(\begin{array}{cc}
\xi_{3} & 0 \\
0 & \xi_{4}
\end{array}\right) M_{34}^{-1}+P \\
& \hat{S}_{2}^{*}=-M M_{12}\left(\begin{array}{cc}
\xi_{1}^{2} & 0 \\
0 & \xi_{2}^{2}
\end{array}\right) M_{12}^{-1}-N M_{12}\left(\begin{array}{cc}
\xi_{1} & 0 \\
0 & \xi_{2}
\end{array}\right) M_{12}^{-1}-P
\end{aligned}
$$


In that case, $\hat{e}_{j}^{m}$ is the solution of the homogeneous system (8), hence zero, so that the boundary operators $S_{j}^{*}$ are perfectly transparent. We can simplify the preceding expressions, which become:

$$
\hat{S}_{1}^{*}=\frac{1}{\operatorname{Re}}\left(\begin{array}{cc}
\frac{\lambda}{|k|}(\lambda+|k|) & -\frac{i k}{|k|} \lambda \\
\frac{i k}{|k|} \lambda & \lambda+|k|
\end{array}\right), \hat{S}_{2}^{*}=\frac{1}{\operatorname{Re}}\left(\begin{array}{cc}
\frac{\lambda}{|k|}(\lambda+|k|) & \frac{i k}{|k|} \lambda \\
-\frac{i k}{|k|} \lambda & \lambda+|k|
\end{array}\right)
$$

An inverse Fourier transform of these operators would lead to non-local pseudodifferential operators, which would not be numerically tractable. In the sequel, we thus want to approximate them by local operators $S_{j}^{a p p}$, that will be called absorbing operators.

\subsection{Approximate boundary conditions}

As indicated above, our present objective is to derive approximations of the $S_{i}^{*} \mathrm{~s}$ that are easy to implement and that lead to a rapid convergence of the Schwarz algorithm. "Easy" means that the $S_{i}^{*}$ s must be local low-order operators, therefore limited to first-order and second-order derivatives. The matrix coefficients in the approximations of the $\hat{S}_{i}^{*}$ s will then be degree two polynomials in $i k$.

\subsubsection{Empirical description of the operators $\hat{S}_{i}^{*}$}

As a first step in this direction, we are going to describe simple properties of $\hat{S}_{1}^{*}$ and $\hat{S}_{2}^{*}$ that will be useful to design relevant approximations.

Sign, symmetry and parity Given the expressions (18), obvious observations are that (i) the anti-diagonal terms are opposite and are odd functions of $k$, and that (ii) the diagonal terms are positive and even functions of $k$. Preserving these properties when building the approximations leads to the following general forms:

$$
\hat{S}_{1}^{a p p}=\left(\begin{array}{cc}
a_{0}-a_{2} k^{2} & -a_{1} i k \\
a_{1} i k & b_{0}-b_{2} k^{2}
\end{array}\right) \text { and } \hat{S}_{2}^{a p p}=\left(\begin{array}{cc}
a_{0}-a_{2} k^{2} & a_{1} i k \\
-a_{1} i k & b_{0}-b_{2} k^{2}
\end{array}\right)
$$

where the $a_{i}$ and $b_{i}$ coefficients are real numbers to be determined, such that $a_{0}-a_{2} k^{2} \geq 0$ and $b_{0}-b_{2} k^{2} \geq 0$.

Orders of magnitude The quantities involved in (18) are $\lambda=\sqrt{\frac{\operatorname{Re}}{\Delta t}+k^{2}}$ and $k$. We have thus to compare $\operatorname{Re} / \Delta t$ to $k^{2}$ to get insights into the orders of magnitude of the different terms in the $\hat{S}_{i}^{*}$ s. The spatial frequencies $k$ that are resolved by the numerical model lie in the interval $\left[\frac{\pi}{H}, \frac{\pi}{\Delta z}\right][7]$. The problem being nondimensional, $H$ is equal to 1 and $1 / \Delta z$ is equal to $N$, the number of grid cells in the $z$-direction. This implies that $\left[k_{\min }, k_{\max }\right]=[\pi, N \pi]$. Considering a "standard" non-dimensional Navier-Stokes numerical model, the CFL ratio $\Delta z / \Delta t$ remains almost constant, independently of $N$, and typically of the order of $10^{0}-10^{2}$. Let 
denote this ratio by $V_{\text {grid }}$. We have then $1 / \Delta t=V_{\text {grid }} / \Delta z=N V_{\text {grid }}$, which leads to

$$
\frac{\operatorname{Re} / \Delta t}{k^{2}} \in\left[\frac{\operatorname{Re} / \Delta t}{k_{\text {max }}^{2}}, \frac{\operatorname{Re} / \Delta t}{k_{\text {min }}^{2}}\right]=\left[\frac{\operatorname{Re} V_{\text {grid }}}{\pi^{2}} \frac{1}{N}, \frac{\operatorname{Re} V_{\text {grid }}}{\pi^{2}} N\right]
$$

In actual applications, Re is of the order of $10^{2}-10^{4}$, and $N$ may vary from a few tens to a few thousands. This implies that $\operatorname{Re} / \Delta t=N \operatorname{Re} V_{\text {grid }}$ is always much larger than $k_{\text {min }}^{2}=\pi^{2}$, and is also in most cases larger than $k_{\text {max }}^{2}=N^{2} \pi^{2}$, except for low Reynolds numbers and relatively high resolution. This means that $\varepsilon=|k| \sqrt{\Delta t / \mathrm{Re}}$ is in most cases a small parameter. We have then

$$
\lambda=\sqrt{\frac{\operatorname{Re}}{\Delta t}\left(1+\varepsilon^{2}\right)}=\sqrt{\frac{\operatorname{Re}}{\Delta t}}\left(1+\frac{\varepsilon^{2}}{2}+O\left(\varepsilon^{4}\right)\right)
$$

which leads to

$$
\hat{S}_{1}, \hat{S}_{2}=\frac{1}{\operatorname{Re}}\left(\begin{array}{cc}
|k|\left(\frac{1}{\varepsilon^{2}}+\frac{1}{\varepsilon}+1+\frac{\varepsilon}{2}\right) & \mp i k\left(\frac{1}{\varepsilon}+\frac{\varepsilon}{2}\right) \\
\pm i k\left(\frac{1}{\varepsilon}+\frac{\varepsilon}{2}\right) & |k|\left(\frac{1}{\varepsilon}+1+\frac{\varepsilon}{2}\right)
\end{array}\right)+o(\varepsilon)
$$

This Taylor expansion highlights the fact that the upper-left coefficient in the $\hat{S}_{i}^{*} \mathrm{~s}$ is often clearly dominant, since it is one order of magnitude larger than the other coefficients. This observation is illustrated in Figure 1, where the modules of the matrix coefficients are plotted for a few values of Re, $V_{\text {grid }}$ and $N$, corresponding to different behaviors (cf Table 1). The future approximations $\hat{S}_{1}^{a p p}$ and $\hat{S}_{2}^{a p p}$ should reflect this property.

Table 1 Description of three numerical cases, spanning different possible values of the model parameters. In each case, the minimal and maximal values of $\varepsilon$ are provided, with $\varepsilon_{\min }=$ $k_{\min } \sqrt{\Delta t / \operatorname{Re}}=\pi / \sqrt{N V_{\text {grid }} \operatorname{Re}}$, and $\varepsilon_{\max }=k_{\max } \sqrt{\Delta t / \operatorname{Re}}=\pi \sqrt{N} / \sqrt{V_{\text {grid }} \operatorname{Re}}$. Case \#2 will be considered for the numerical simulations (see Section 4).

\begin{tabular}{ccccccc}
\hline & Re & $V_{\text {grid }}$ & $N$ & $\Delta t$ & $\varepsilon_{\min }$ & $\varepsilon_{\max }$ \\
\hline Case 1 & $10^{4}$ & 10 & 256 & $3.910^{-4}$ & $610^{-4}$ & 0.16 \\
Case 2 & $10^{3}$ & 1 & 64 & $1.610^{-2}$ & 0.01 & 0.79 \\
Case 3 & 500 & 1 & 1024 & $9.810^{-4}$ & $410^{-3}$ & 4.50 \\
\hline
\end{tabular}
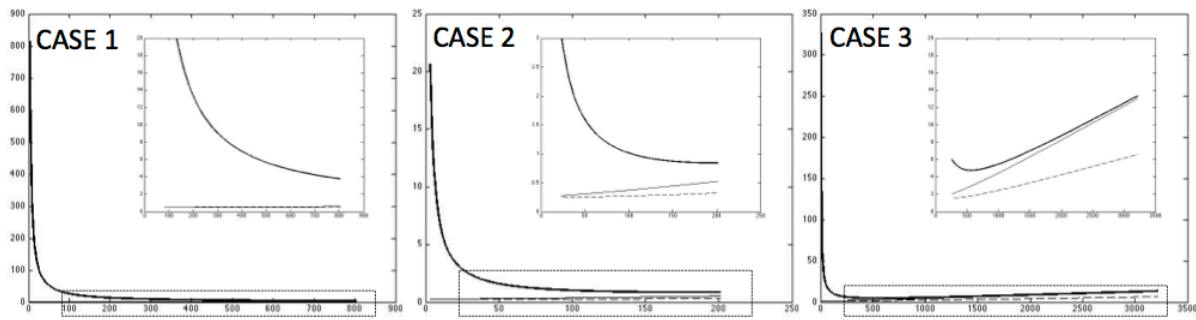

Fig. 1 Comparison of the modules of the coefficients in $\hat{S}_{1}^{*}$ and $\hat{S}_{2}^{*}$ as a function of $k$, with additional zooms away from $k_{\min }$ (corresponding to the dashed rectangles). Thick solid line: $\left|\hat{S}_{i}(1,1)\right|$; dashed line: $\left|\hat{S}_{i}(1,2)\right|=\left|\hat{S}_{i}(2,1)\right|$; thin solid line: $\left|\hat{S}_{i}(2,2)\right|$. 
Table 2 Summary of the different approximations of $\hat{S}_{1}^{*}$ and $\hat{S}_{2}^{*}$

\begin{tabular}{|c|c|c|}
\hline \multicolumn{3}{|c|}{ expansion at order $1 / \varepsilon^{2}$} \\
\hline $\begin{array}{c}\text { degree } 0 \\
\text { polynomial }\end{array}$ & & $\begin{array}{c}\text { degree } 2 \\
\text { polynomial }\end{array}$ \\
\hline$\hat{S}_{i}^{-2,0}=\left(\begin{array}{cc}a_{0} & 0 \\
0 & 0\end{array}\right)$ & & $\hat{S}_{i}^{-2,2}=\left(\begin{array}{cc}a_{0}-a_{2} k^{2} & 0 \\
0 & 0\end{array}\right)$ \\
\hline$a_{0} \geq 0$ & & $a_{0}-a_{2} k^{2} \geq 0$ on $\left[k_{\min }, k_{\max }\right]$ \\
\hline \multicolumn{3}{|c|}{ expansion at order $1 / \varepsilon$} \\
\hline $\begin{array}{c}\text { degree } 0 \\
\text { polynomial }\end{array}$ & $\begin{array}{l}\text { degree } 1 \\
\text { polynomial }\end{array}$ & $\begin{array}{c}\text { degree } 2 \\
\text { polynomial }\end{array}$ \\
\hline$\hat{S}_{i}^{-1,0}=\left(\begin{array}{cc}a_{0} & 0 \\
0 & b_{0}\end{array}\right)$ & $\hat{S}_{i}^{-1,1}=\left(\begin{array}{cc}a_{0} & \mp a_{1} i k \\
\pm a_{1} i k & b_{0}\end{array}\right)$ & $\hat{S}_{i}^{-1,2}=\left(\begin{array}{cc}a_{0}-a_{2} k^{2} & \mp a_{1} i k \\
\pm a_{1} i k & b_{0}-b_{2} k^{2}\end{array}\right)$ \\
\hline$a_{0}, b_{0} \geq 0$ & $a_{0}, b_{0} \geq 0$ & $a_{0}-a_{2} k^{2}, b_{0}-b_{2} k^{2} \geq 0$ on $\left[k_{\min }, k_{\max }\right]$ \\
\hline
\end{tabular}

\subsubsection{A series of approximations}

As indicated above, a general admissible form for the approximate operators $\hat{S}_{i}^{a p p}$ is given by (19). Rather than looking globally for optimal values of the five coefficients $a_{i}$ and $b_{i}$, we can propose a series of approximations of increasing complexity, based on the Taylor expansion (21).

- At lowest order in $\varepsilon$, i.e. $1 / \varepsilon^{2}$, the upper-left coefficient is the only non zero element in $\hat{S}_{i}^{a p p}$. It can be represented either by a constant positive value $a_{0}$ (therefore avoiding second order derivatives in the approximate boundary conditions), or by a degree two polynomial $a_{0}-a_{2} k^{2}$, positive for $k \in\left[k_{\min }, k_{\max }\right]$. The two corresponding approximations of $\hat{S}_{i}^{*}$ are denoted $\hat{S}_{i}^{-2,0}$ and $\hat{S}_{i}^{-2,2}$ respectively (see Table 2).

- At order -1 in $\varepsilon$, the expansion (21) indicates that the four elements of $\hat{S}_{i}^{*}$ must be taken into account. This means that the $\hat{S}_{i}^{a p p}$ s are given either by the full expression (19) further denoted $\hat{S}_{i}^{-1,2}$, or by its simplified version $\hat{S}_{i}^{-1,1}$ where $a_{2}=b_{2}=0$ if one wants to avoid second order derivatives, or by an even more simplified version $\hat{S}_{i}^{-1,0}$ where $a_{1}=a_{2}=b_{2}=0$ if one only allows degree zero polynomials.

This series of approximate operators is summarized in Table 2.

\subsubsection{Theoretical convergence performances}

Our goal is to design efficient interface operators, that will lead to a fast convergence of the Schwarz algorithm. As seen in section 2.3, the convergence rate is given by the spectral radius of the amplification matrix (16). The analytical expressions of the eigenvalues of this $2 \times 2$ matrix can be determined for the different approximate boundary operators, as functions of the coefficients $a_{i}$ and $b_{i}$. However these expressions, that we obtained using a symbolic computation software, 
are quite long and complicated. Therefore we will not give their detailed expression (except in the very simple case of approximation $\hat{S}_{i}^{-2,0}$ with no overlap), but rather comment on the smallest values of the spectral radius $\rho$ defined in (17) that can be obtained after minimization w.r.t. the $a_{i}$ and $b_{i}$ coefficients.

Approximations at order -2 in $\varepsilon$ For the approximation $\hat{S}_{i}^{-2,0}=\left(\begin{array}{cc}a_{0} & 0 \\ 0 & 0\end{array}\right)$, and in the non overlapping case, the eigenvalues are

$$
1 \text { and }\left(\frac{\lambda\left(\lambda^{3}-k^{2}|k|\right)+a_{0} \operatorname{Re}|k|\left(k^{2}-\lambda^{2}\right)}{-\lambda\left(\lambda^{3}-k^{2}|k|\right)+a_{0} \operatorname{Re}|k|\left(k^{2}-\lambda^{2}\right)}\right)^{2}
$$

The eigenvalues for the approximation $\hat{S}_{i}^{-2,2}$ are of course the same, but replacing $a_{0}$ by $a_{0}-a_{2} k^{2}$.

An important result here is that one of the eigenvalues is equal to 1 , which indicates that the Schwarz algorithm will not converge in this case where the subdomains do not overlap. The explanation for this is actually quite simple. As a matter of fact, when using approximation $\hat{S}_{i}^{-2,0}$, the conditions occurring in the Schwarz algorithm at the common interface are

$$
\left\{\begin{array}{l}
-\frac{1}{\operatorname{Re}} \frac{\partial u_{1}^{m}}{\partial x}+p_{1}^{m}-a_{0} u_{1}^{m}=-\frac{1}{\operatorname{Re}} \frac{\partial u_{2}^{m-1}}{\partial x}+p_{2}^{m-1}-a_{0} u_{2}^{m-1} \\
-\frac{1}{\operatorname{Re}} \frac{\partial w_{1}^{m}}{\partial x}=-\frac{1}{\operatorname{Re}} \frac{\partial w_{2}^{m-1}}{\partial x}
\end{array}\right.
$$

and

$$
\left\{\begin{array}{l}
\frac{1}{\operatorname{Re}} \frac{\partial u_{2}^{m}}{\partial x}-p_{2}^{m}-a_{0} u_{2}^{m}=\frac{1}{\operatorname{Re}} \frac{\partial u_{1}^{m-1}}{\partial x}-p_{1}^{m-1}-a_{0} u_{1}^{m-1} \\
\frac{1}{\operatorname{Re}} \frac{\partial w_{2}^{m}}{\partial x}=\frac{1}{\operatorname{Re}} \frac{\partial w_{1}^{m-1}}{\partial x}
\end{array}\right.
$$

Due to the change of sign between (23a) and (24a), the first condition will lead to a change from one iteration to the other. But, conversely, the second conditions (23b) and (24b) are similar for both subdomains. Therefore, in the case of a non overlapping configuration, the quantity $\partial w / \partial x$ does not evolve, which prevents the algorithm from converging. If the approximation $\hat{S}_{i}^{-2,2}$ is used instead of $\hat{S}_{i}^{-2,0}$, the conditions (23b) and (24b) remain unchanged, hence the non convergence. In summary, these approximations $\hat{S}_{i}^{-2,0}$ and $\hat{S}_{i}^{-2,2}$ cannot be used without an overlap.

In the case of overlapping subdomains $(\delta>0)$, the spectral radius can however become less than 1 . As can be observed on Figure 2 for the approximation $\hat{S}_{i}^{-2,0}$, the minimal value of $\rho(\hat{H})$ is always smaller than 0.5 , so that we can expect a fast convergence of the Schwarz algorithm. Figure 2 is plotted for $a_{0} \geq 0$, consistently with the constraint expressed in Table 2 . However we have also verified that choosing $a_{0}<0$ leads to poorer results, as will be showed for instance in Figure 3. Among our three sets of parameter values, the more favorable one is case \#2, but this cannot be directly evidenced by the analytical expression of $\rho(\hat{H})$ since its dependency with respect to $a_{0}, \delta$ and the parameters listed in Table 1 is way too 
complicated. Note that increasing the overlap from $\delta=\Delta x$ to $\delta=2 \Delta x$ slightly changes the optimal value of $a_{0}$, and significantly improves the spectral radius at a low computational cost.

It is interesting to compare the performance of these interface conditions w.r.t. simple Dirichlet conditions $\left(\mathbf{v}_{j}^{m}, p_{j}^{m}\right)=\left(\mathbf{v}_{j^{\prime}}^{m-1}, p_{j^{\prime}}^{m-1}\right)$ on $\Gamma_{j}$. In this latter case, the recursive formula for the errors (15) is replaced by

$$
\gamma_{12}^{m}=\hat{H}_{d i r} \gamma_{12}^{m-2} \quad \text { with } \hat{H}_{d i r}=e^{-\xi_{12} \delta} M_{12}^{-1} M_{34} e^{\xi_{34} \delta} M_{34}^{-1} M_{12}
$$

The spectral radius of $\hat{H}_{d i r}$ can be easily computed, and we can see (Table 3 , first raw) that it never becomes smaller than 0.95 for $\delta=\Delta x$ and 0.85 for $\delta=2 \Delta x$. Approximation $\hat{S}_{i}^{-2,0}$ is thus a very strong improvement w.r.t. a simple DirichletDirichlet approach.

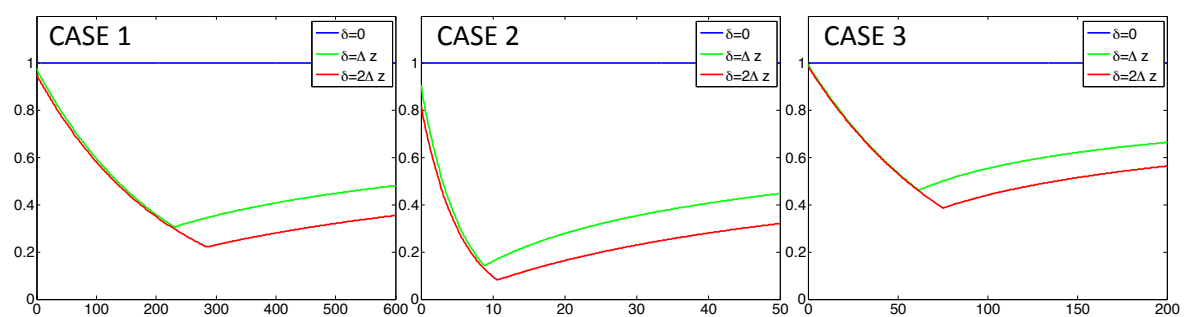

Fig. 2 Illustration of the theoretical convergence rate for approximation $S^{-2,0}$ : spectral radius $\rho(\hat{H})$ as a function of $a_{0}$ for $\delta=0$ (blue curves), $\delta=\Delta x$ (green curves) and $\delta=2 \Delta x$ (red curves). The related configurations are detailed in Table 1.

Results obtained with the approximation $\hat{S}_{i}^{-2,2}$ are displayed in Figure 3. For the sake of clarity, we only consider case \#1 here, but the other cases were also performed, leading to similar conclusions. First of all, note that the horizontal section $a_{2}=0$ of course exactly corresponds to the spectral radius displayed in Figure 2 (left panel) for the approximation $\hat{S}_{i}^{-2,0}$. Moreover, there is clearly an "area of convergence" in the $\left(a_{0}, a_{2}\right)$ plane, where $\rho(\hat{H})<1$. This area is fully consistent with the empirical constraint $a_{0}-a_{2} k^{2} \geq 0$ for $k \in\left[k_{\min }, k_{\max }\right]$ proposed in Section 3.2.1. As a matter of fact, this constraint corresponds to the intersection of the two half-planes $a_{0}-a_{2} k_{\min }^{2} \geq 0$ and $a_{0}-a_{2} k_{\max }^{2} \geq 0$, and the area of convergence matches almost exactly this intersection for $\delta=\Delta x$ and $\delta=2 \Delta x$. Finally, it appears that adding the second degree term $a_{2} k^{2}$ in the polynomial approximation of the leading coefficient of $\hat{S}_{i}$ has almost no influence on the convergence properties within the area of convergence. As a matter of fact, the isolines of $\rho(\hat{H})$ in this region are almost parallel to the vertical axis. Actually, its minimal value is 0.3048 , obtained for $\left(a_{0}^{o p t} ; a_{2}^{o p t}\right)=\left(230.6 ; 3.610^{-4}\right)$, while the corresponding value of the convergence rate for approximation $\hat{S}_{i}^{-2,0}$ was 0.3059 , obtained for almost the same value $a_{0}^{o p t}=229.9$. This extremely weak influence of the $a_{2}$ term is observed in the 3 cases (see Table 3 ).

We will come back in section 4 to the convergence rate observed in actual numerical simulations. 

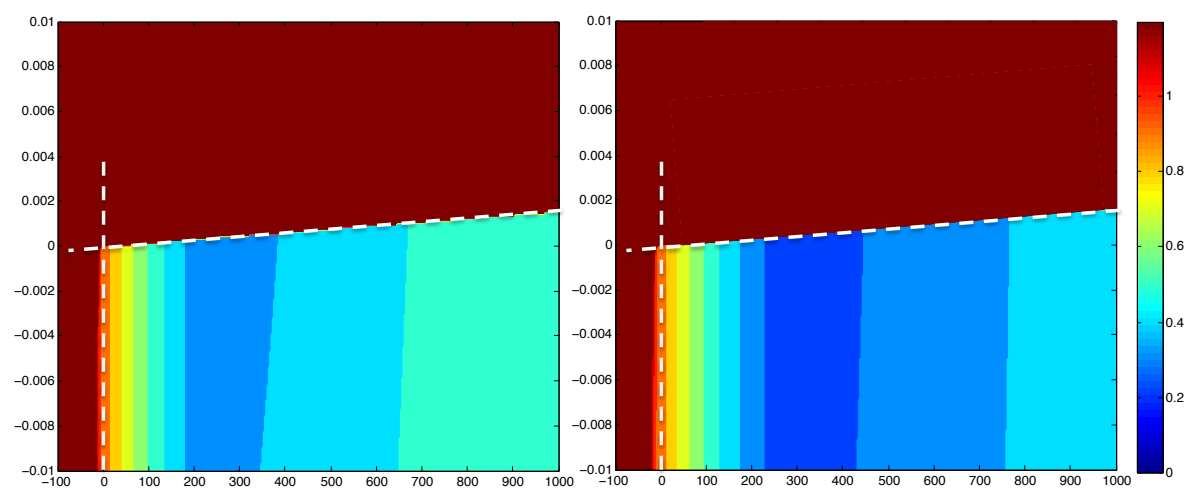

Fig. 3 Illustration of the theoretical convergence rate for approximation $S^{-2,2}$ in the case \#1. Spectral radius $\rho(\hat{H})$ as a function of $a_{0}$ (x-axis) and $a_{2}$ (y-axis) for $\delta=\Delta x$ (left) and $\delta=2 \Delta x$ (right). The dashed lines correspond to $a_{0}-a_{2} k_{\min }^{2}=0$ and $a_{0}-a_{2} k_{\max }^{2}=0$.

Approximations at order -1 in $\varepsilon$ In order to obtain the expressions of the eigenvalues of the amplification matrices corresponding to approximations $\hat{S}_{i}^{-1, n}(n=$ $0,1,2)$, it is sufficient to get them for $\hat{S}_{i}^{-1,1}$. The eigenvalues for $\hat{S}_{i}^{-1,0}$ (respectively $\hat{S}_{i}^{-1,2}$ ) can then obtained by setting $a_{1}=0$ (respectively by replacing $a_{0}$ and $b_{0}$ by $a_{0}-a_{2} k^{2}$ and $b_{0}-b_{2} k^{2}$ ). As for the preceding approximations at order -2 , we obtained these expressions using a symbolic computation software.

As expected, approximation at order -1 performs clearly better than approximation at order -2 . In the nonoverlapping case, 1 is no longer an eigenvalue, and the convergence rate may become smaller than 1 .

- For approximation $S^{-1,0}$, the Schwarz algorithm without overlap converges for a wide range of values of $a_{0}$ and $b_{0}$, including the quadrant $a_{0}, b_{0}>0$, as can be seen in Figure 4 (note that, if $a_{0}$ and $b_{0}$ both become large enough, the interface conditions reduce to a simple Dirichlet-Dirichlet case, as can be seen in equations (12)-(13)). The optimal spectral radius reached with this approximation $S^{-1,0}$ is rather efficient: $\rho_{\text {opt }}=0.733,0.381$ and 0.595 for the three test cases respectively (Table 3 ). Note that the ratio $a_{0}^{o p t} / b_{0}^{o p t}$ between the optimal coefficients is fully consistent with the theoretical optimal ratio $\hat{S}_{11}^{*}(k) / \hat{S}_{22}^{*}(k)=\lambda /|k|=\sqrt{1+R e /\left(\Delta t k^{2}\right)}$, which is approximately $O\left(10^{1}-\right.$ $\left.10^{2}\right), O\left(10^{0}-10^{1}\right)$ and $O\left(10^{0}-10^{1}\right)$ respectively for the 3 cases (Figure 4 and Appendix).

- Adding a small overlap clearly enhances the convergence, which becomes extremely fast in our first two test cases.

- The addition of the extra diagonal terms $\pm a_{1} i k$ (approximation $\hat{S}_{i}^{-1,1}$ ) slightly improves the spectral radius, at most by $10-15 \%$ in the most favorable case (Table 3). Note that, once again, the ratio $a_{0}^{o p t} / a_{1}^{o p t}$ observed in our test cases is generally fully consistent with the corresponding theoretical ratio $\left|k \hat{S}_{11}^{*}(k) / \hat{S}_{12}^{*}(k)\right|=\lambda+|k|$, which is $O\left(10^{3}\right), O\left(10^{2}\right)$ and $O\left(10^{3}\right)$ respectively for the 3 cases (see Appendix).

- Considering $\hat{S}_{i}^{-1,2}$, i.e. adding the $a_{2}$ and $b_{2}$ terms, makes the optimization much more complex, since one has now 5 parameters to consider. We did not 


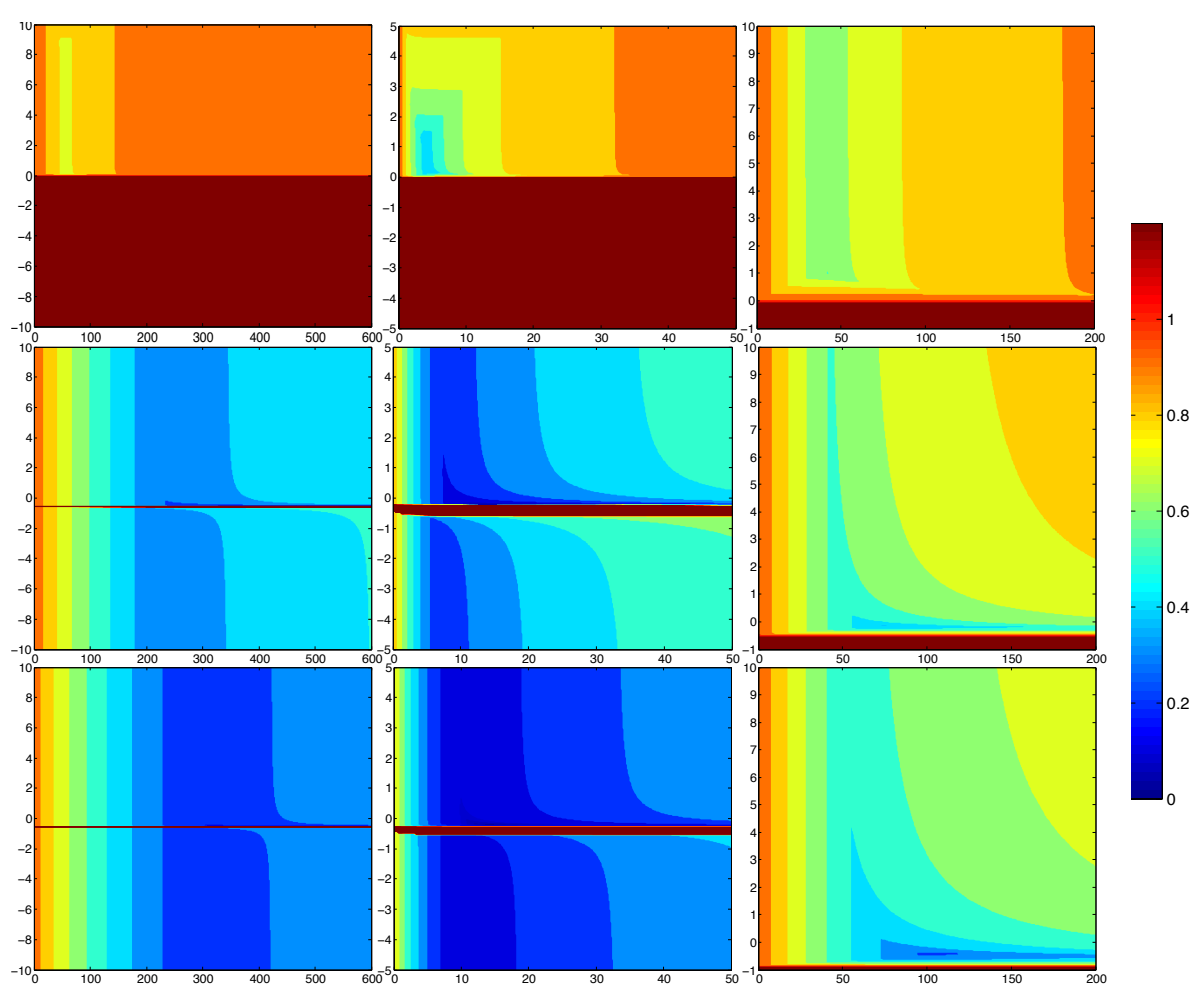

Fig. 4 Illustration of the theoretical convergence rate for approximation $S^{-1,0}$. Top line: spectral radius $\rho(\hat{H})$ as a function of $a_{0}$ (x-axis) and $b_{0}$ (y-axis) for $\delta=0$. Middle line: same configuration with $\delta=\Delta x$. Bottom line: same configuration with $\delta=2 \Delta x$. The 3 columns correspond to the 3 configurations detailed in Table 1.

perform a full optimization, but our attempts to add those two degrees of freedom in the vicinity of values $a_{0}, b_{0}$ and $a_{1}$ that are optimal for $\hat{S}_{i}^{-1,1}$ did not lead to any significant decrease in $\rho_{\text {opt }}$ (not shown). This seems to confirm the very weak influence of the $a_{2}$ term in approximation $S^{-2,2}$ w.r.t. $S^{-2,0}$ that we observed previously.

Discussion From the preceding investigations, we can already draw some first conclusions:

- Approximations $\hat{S}_{i}^{-k, 1}$ and $\hat{S}_{i}^{-k, 2}$ bring very few improvement w.r.t. $\hat{S}_{i}^{-k, 0}$ $(k=-2,-1)$. On the opposite, $\hat{S}_{i}^{-1,0}$ leads to much better results than $\hat{S}_{i}^{-2,0}$. The approximation $\hat{S}_{i}^{-1,0}$ appears thus as a good overall compromise.

- If ones does not want the subdomains to overlap, then $\hat{S}_{i}^{-1, p}$ must be used since $\hat{S}_{i}^{-2, p}$ cannot lead to convergence. On the opposite, a small overlap, if affordable, generally accelerates very much the convergence.

- The different forms of matrix $\hat{S}_{i}^{a p p}$ were proposed using a Taylor expansion that is valid if $\varepsilon$ is small. However, even if this is not the case, these matrix forms can still be used and their coefficients can be optimized in order to minimize $\rho(\hat{H})$ efficiently. Test case \#3 is an illustration of such a situation. 
Table 3 Optimal value of $\rho(\hat{H})$ as a function of the overlap for the different approximations. The optimal values for coefficients $a_{0}, b_{0}$ and $a_{1}$ are indicated in Appendix.

\begin{tabular}{c|ccc|ccc|ccc|} 
& \multicolumn{3}{|c|}{ Case 1} & \multicolumn{3}{c|}{ Case 2} & \multicolumn{3}{c|}{ Case 3} \\
& $\delta=0$ & $\Delta x$ & $2 \Delta x$ & $\delta=0$ & $\Delta x$ & $2 \Delta x$ & $\delta=0$ & $\Delta x$ & $2 \Delta x$ \\
\hline Dir-Dir & 1. & .978 & .955 & 1. & .951 & .864 & 1. & .999 & .998 \\
$\hat{S}_{i}^{-2,0}$ & 1. & .306 & .221 & 1. & .142 & .081 & 1. & .462 & .386 \\
$\hat{S}_{i}^{-2,2}$ & 1. & .305 & .220 & 1. & .140 & .081 & 1. & .461 & .386 \\
$\hat{S}_{i}^{-1,0}$ & .733 & .067 & .038 & .381 & .040 & .008 & .595 & .394 & .265 \\
$\hat{S}_{i}^{-1,1}$ & .650 & .066 & .038 & .332 & .034 & .008 & .592 & .356 & .244 \\
\hline
\end{tabular}

\section{Numerical simulations}

The objective of this section is to provide a numerical illustration of the theoretical results presented in Section 3. We first set the numerical framework (time-space finite differences for the lid-driven cavity, see [2] for details), and present in the sequel the impact of our optimized boundary conditions on the domain decomposition method used for the driven cavity.

\subsection{Numerical framework}

Driven cavity The driven cavity problem is well known, since it has been investigated by many authors (see for instance [2] and the numerous references herein). The computational domain is a vertical 2 -D square domain $\Omega=(0,1) \times(0,1)$, in which the incompressible Navier-Stokes equations (1) are solved, with no-slip boundary conditions on 3 sides of the cavity and a driving velocity on the top of the cavity:

$$
\left\{\begin{array}{l}
\mathbf{v}(0, z, t)=\mathbf{v}(1, z, t)=\mathbf{v}(x, 0, t)=\mathbf{0}, \quad 0 \leq x, z \leq 1, t>0 \\
\mathbf{v}(x, 1, t)=(1,0), \quad 0 \leq x \leq 1, t>0
\end{array}\right.
$$

The simulation is started from rest: $\mathbf{v}(x, z, 0)=\mathbf{0}, 0 \leq x, z \leq 1$.

Discretization method Discretization in time is performed using a first order Euler scheme, leading to the semi-discrete system (2). Discretization in space is performed on a uniform staggered grid (see Figure 5) using a third order scheme for the nonlinear term, as described in [2]. Numerical experiments that will be presented in the following were performed at $\operatorname{Re}=10^{4}$, with $\Delta x=\Delta z=1 / 256$, $\Delta t=\Delta z / 10$ which corresponds to the case \# 1 in Section 3 and Table 1 . For those values of the parameters, the stationary flow exhibits a large primary clockwise vortex (see Figure 5), with secondary vortices in the corners (counter clockwise or clockwise, depending on the order in the well-known vortices cascade). Our numerical model was validated by comparison to corresponding results presented in the literature (e.g. $[2,6])$. 

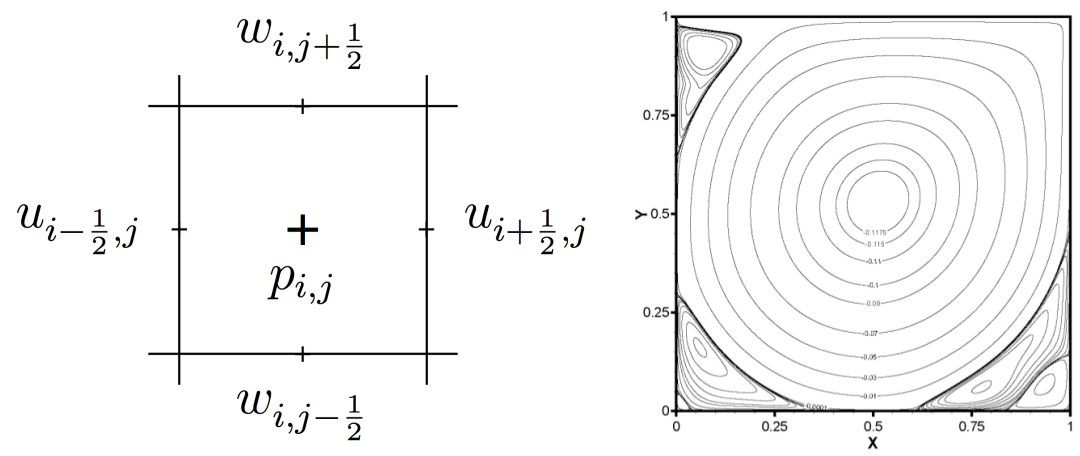

Fig. 5 Left: a staggered cell. Right: typical solution of the lid-driven cavity problem at $\mathrm{Re}=$ $10^{4}$.

4.2 Optimized boundary conditions discretized

With the discretization method introduced in Section 4.1, we now illustrate the efficiency of the Schwarz iterative method equipped with the interface conditions introduced in Section 3. For the sake of succinctness, we only consider the test case \#1, with Dirichlet-Dirichlet, $S^{-2,0}$ and $S^{-1,0}$ boundary conditions.

While the Dirichlet-Dirichlet conditions are quite classical and do not require any additional numerical description, let us give a few details regarding the way interface conditions $S^{-2,0}$ (namely (23) and (24)) are discretized (processing conditions $S^{-1,0}$ is similar). In particular for equation (23a) we consider the following ${ }^{2}$ :

$$
\begin{aligned}
-\frac{1}{\operatorname{Re}} \frac{u_{I+1 / 2, j}^{1}-u_{I-1 / 2, j}^{1}}{\Delta x}+p_{I, j}^{1} & -a_{0} u_{I+1 / 2, j}^{1} \\
& =-\frac{1}{\operatorname{Re}} \frac{u_{I+3 / 2, j}^{2}-u_{I+1 / 2, j}^{2}}{\Delta x}+p_{I+1, j}^{2}-a_{0} u_{I+1 / 2, j}^{2}+A
\end{aligned}
$$

where:

- $i=I+1 / 2$ holds for the interface location, where both $u_{I+1 / 2, j}^{1}$ and $u_{I+1 / 2, j}^{2}$ are defined,

- $A=-\Delta x\left[f_{I+1 / 2, j}+\frac{D_{z}^{2} u_{I+1 / 2, j}^{1}+D_{z}^{2} u_{I+1 / 2, j}^{2}}{2 \operatorname{Re}}+\frac{u_{I+1 / 2, j}^{1}+u_{I+1 / 2, j}^{2}}{2 \Delta t}\right]$,

- $D_{z}^{2} u_{i, j}$ denotes $\frac{u_{i, j+1}-2 u_{i, j}+u_{i, j-1}}{\Delta z^{2}}$.

It is clear that $A$ is of order $\Delta x$, so that equation (26) is a consistant approximation of equation (23a). The $A$ term, which may look useless at first glance, is chosen so that equation (26) is consistent (after convergence) with the monodomain approximation of the first equation (3) on the whole interface, namely:

$$
\frac{u_{I+1 / 2, j}}{\Delta t}-\frac{D_{x}^{2} u_{I+1 / 2, j}+D_{z}^{2} u_{I+1 / 2, j}}{\operatorname{Re}}+D_{x} p_{I+1 / 2, j}=f_{I+1 / 2, j}, \quad \forall j,
$$

2 For the sake of readability, the Schwarz indices $m$ and $m-1$ have been removed, but $u^{1}$ should be read as $u^{1, m}$, and $u^{2}$ as $u^{2, m-1}$ as in (23a). In the other case $\left(u^{1}=u^{1, m-1}\right.$ and $\left.u^{2}=u^{2, m}\right)$, we would obtain the discretized version of $(24 \mathrm{a})$. 
where $D_{x} p_{i+1 / 2, j}=\frac{p_{i+1, k}-p_{i, j}}{\Delta x}$ and $D_{x}^{2} u_{I+1 / 2, j}=\frac{u_{I+3 / 2, j}-2 u_{I+1 / 2, j}+u_{I-1 / 2, j}}{\Delta x^{2}}$.

\subsection{Numerical results}

Our numerical results were obtained thanks to the Schwarz method (in the multiplicative case) implemented with the aforementioned interface boundary conditions in the case of two subdomains. We illustrate the convergence of the iterative method on one single time step, at time $t=1 .^{3}$

As expected (see discussion in Section 3), none of the Dirichlet-Dirichlet nor $S^{-k, 0}$ interface conditions makes the DDM algorithm converge when $\delta=0$ (no overlap). For $\delta>0$ (overlapping domains), the numerical results are almost identical to the theoretical values as far as the Dirichlet-Dirichlet condition is concerned (compare the first line in Table 4 with the first line in Table 3). Moreover the numerical results obtained with $S^{-2,0}$ are in very good accordance with theoretical results:

- the shape of the convergence rate with respect to coefficient $a_{0}$ (Figure 6) is very similar to the theoretical one (Figure 2, left panel),

- the optimal value $a_{0}^{*}$ is of the same order of magnitude in both numerical and theoretical cases.

As could be expected, the exact values of $a_{0}^{*}$ and of the corresponding numerical convergence rates are not identical (compare the second line in Table 3 with the second line in Table 4), but this can be explained by the hypotheses that were made to build the theoretical analysis (infinite domain) and by the discretization of continuous equations. As expected also from the theoretical results, the obtained convergence rates compare very favorably to the ones obtained with DirichletDirichlet interface conditions, which supports the use of $S^{-2,0}$ conditions rather than Dirichlet-Dirichlet conditions as soon as subdomains overlap.

Numerical results obtained with $S^{-1,0}$ are also rather consistent with theoretical ones. Although we did not extensively explore all possible values for $\left(a_{0}, b_{0}\right)$, sections in the $\left(a_{0}, b_{0}\right)$ plane (Figure 7$)$ indicate a V-shaped behavior of $\rho(\hat{H})$ as a function of $a_{0}$, and a more complex shape as a function of $b_{0}$. These shapes are consistent with the theoretical behavior plotted in Figure 4 (second row, left column), although less contrasted.

Table 4 Optimal value of $\rho(\hat{H})$ as a function of the overlap in our numerical experiments. The optimal values for coefficient $a_{0}$ are indicated in the last line.

\begin{tabular}{c|ccc|} 
& $\delta=0$ & $\Delta x$ & $2 \Delta x$ \\
\hline Dir-Dir & 1. & .950 & 922 \\
$\hat{S}^{-2,0}$ & 1. & .514 & .425 \\
\hline$a_{0}^{*}$ & N/A & 243.5 & 300.6
\end{tabular}

\footnotetext{
3 The time steps from $t=0$ to $t=1$ have been previously computed with the monodomain algorithm in order to avoid starting from a zero initial solution, which could hide numerical evidences.
} 


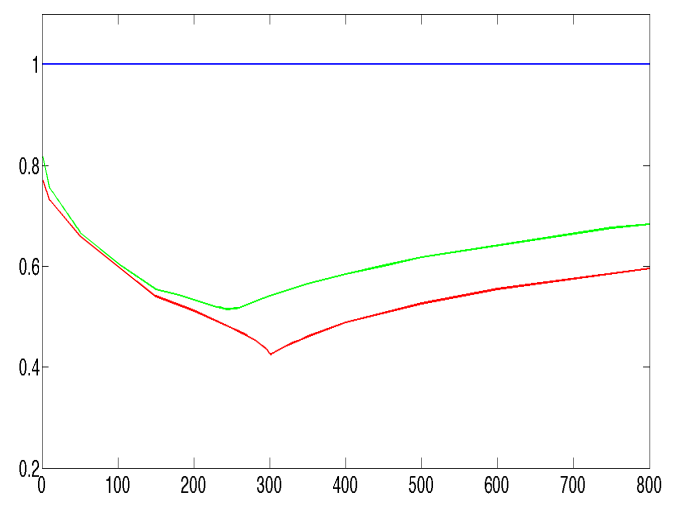

Fig. 6 Numerical convergence rate for approximation $S^{-2,0}$ for $\delta=0$ (blue), $\delta=\Delta x$ (green) and $\delta=2 \Delta x$ (red). The related configuration is Test Case \#1 in Table 1.

Remark 1 Note that another possible use of the Schwarz domain decomposition algorithm is no longer as an iterative solver, but as a preconditioner for a Krylov method, such as GMRES for instance. It has indeed been noticed in many papers (e.g. [7]) that the convergence is more efficient that way. We thus implemented a version of our test case in such a way, similar to case \#1 except that $N=64$. Our results are summarized in Table 5 (to compare both methods, we transformed the number of subdomain resolutions in GMRES into an equivalent convergence rate). Consistently with previous studies, we observed that using the Dirichlet-Dirichlet Schwarz method as a preconditioner for GMRES leads to a much more efficient convergence rate than solving directly with the Dirichlet-Dirichlet classical algorithm (6): $\rho=0.24$ instead of $\rho=0.87$. More surprisingly, using the Schwarz method with $S^{-2,0}$ conditions as a preconditioner does not improve the convergence rate any longer. Although the hierarchy in the performances of interface conditions is generally conserved when skipping from using Schwarz as a solver to using Schwarz as a preconditioner, an example of such a similar result was obtained recently in the context of an advection-diffusion problem [11]. This result should thus be studied more carefully, in particular in terms of matrix spectra, and should be complemented by additional results with other interface conditions and other physical configurations. This is however out of the scope of the present paper, and will be the subject of future investigations.

Table 5 Comparison of the convergence rate of the Schwarz method, either used as an iterative solver or as a preconditioner for a GMRES solver $(\delta=\Delta x)$.

\begin{tabular}{l|cc|} 
& Dir-Dir & $S^{-2,0}$ \\
\hline Schwarz as an iterative solver & 0.87 & 0.41 \\
Schwarz as a preconditioner for GMRES & 0.24 & 0.50
\end{tabular}



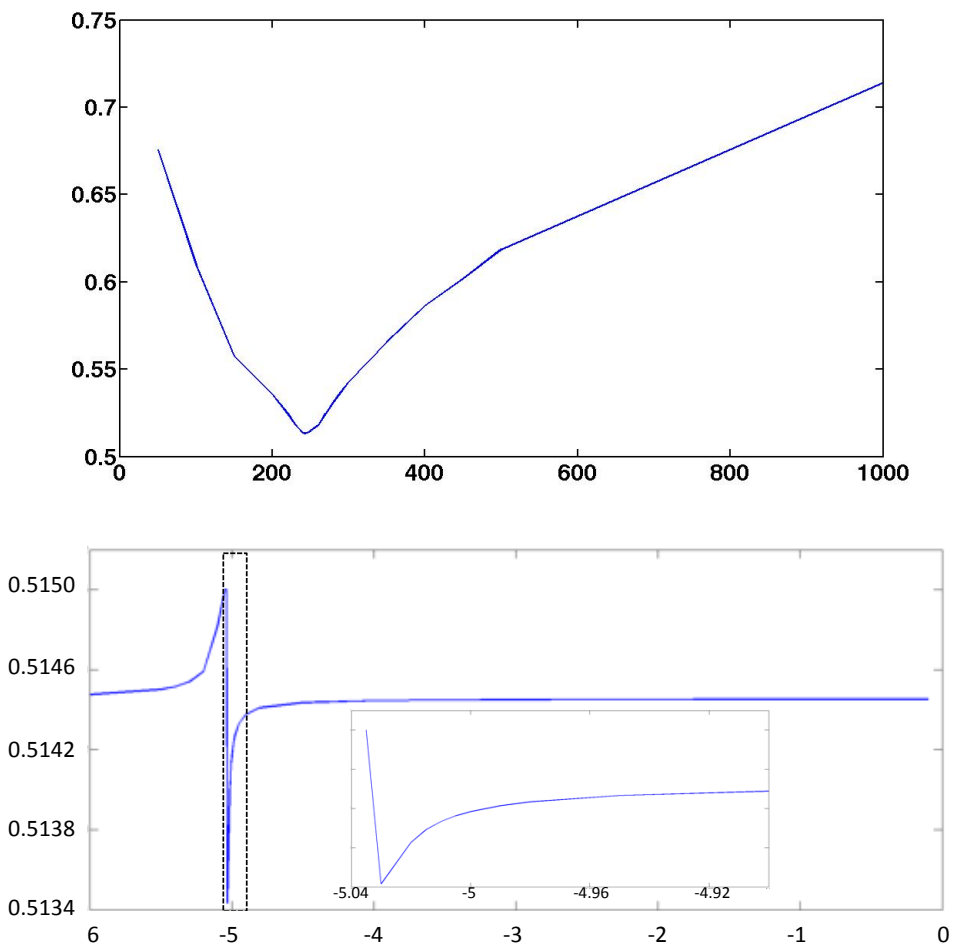

Fig. 7 Numerical convergence rate for approximation $S^{-1,0}$. The related configuration is Test Case \#1 in Table 1. Top: convergence as a function of $a_{0}$ (with $b_{0}=-5.03$ ). Bottom: convergence as a function of $b_{0}$ (with $a_{0}=243.5$ ). The additional zoom corresponds to the dashed rectangle.

\section{Concluding remarks}

In order to complement the preceding study, we would like to point out two additional aspects that have not been discussed yet.

\subsection{Two-sided conditions}

Up to now, the values of the coefficients $a_{i}$ and $b_{i}$ are identical in $\hat{S}_{1}^{a p p}$ and $\hat{S}_{2}^{a p p}$. However it is possible to switch from such so called one-sided conditions to twosided ones, in which these coefficients may have different values in $\hat{S}_{1}^{a p p}$ and $\hat{S}_{2}^{a p p}$. We have tested this alternative for the simplest case $\hat{S}^{-2,0}$, i.e. considering

$$
\hat{S}_{1}^{-2,0}=\left(\begin{array}{cc}
a_{0} & 0 \\
0 & 0
\end{array}\right) \quad \text { and } \quad \hat{S}_{2}^{-2,0}=\left(\begin{array}{cc}
a_{0}^{\prime} & 0 \\
0 & 0
\end{array}\right)
$$

The two-sided spectral radius is plotted in Figure 8. Its optimal value $\rho_{\text {opt }}\left(a_{0}, a_{0}^{\prime}\right)$ is $30-35 \%$ smaller than the corresponding optimal one-sided spectral radius $\rho_{\text {opt }}\left(a_{0}\right)$ 
(located on the diagonal of each plot), for each of our three test cases (see Appendix). This improvement is quite significant.
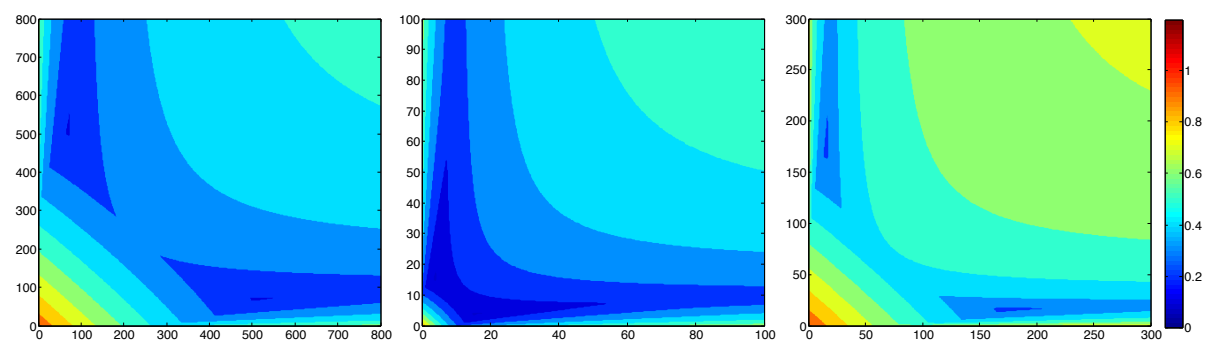

Fig. 8 Approximation $\hat{S}^{-2,0}$ in the two-sided case: spectral radius $\rho(\hat{H})$ as a function of $a_{0}$ ( $x$-axis) and $a_{0}^{\prime}$ (y-axis) for $\delta=\Delta x$ in the case \#1. The 3 panels correspond to the 3 configurations detailed in Table 1.

However this two-sided approach doubles the number of degrees of freedom (dof) in the $\hat{S}_{i}^{a p p}$ operators, which makes the optimization harder. The question is then to determine which approximation leads to the best convergence properties for a given number of dof. If we limit the optimization to 2 dof, which is reasonable for the optimization in practical cases, we have then to compare two-sided approximation $\hat{S}^{-2,0}$ (where dof are $a_{0}$ and $a_{0}^{\prime}$ ) with one-sided approximation $\hat{S}^{-1,0}$ (where dof are $a_{0}$ and $\left.b_{0}\right)$. For our test cases, there is no fully clear conclusion, since one-sided $\hat{S}^{-1,0}$ leads to much better spectral radii than two-sided approximation $\hat{S}^{-2,0}$ in cases \#1 and \#2, but not in case \#3 (see Appendix). However, considering that $\hat{S}^{-1,0}$ does not require any overlap to converge, this latter approximation probably remains the best overall compromise.

\subsection{Symmetric tensor}

As mentioned previously, the stress tensor that we used in Section 2.1 to derive the general form of the interface conditions was the non symmetric form $\boldsymbol{\sigma}=1 / \operatorname{Re} \nabla \boldsymbol{v}-p$ Id. However we could also have chosen the symmetric one $\boldsymbol{\sigma}=1 /(2 \mathrm{Re})\left(\nabla \boldsymbol{v}+\nabla \boldsymbol{v}^{T}\right)-p$ Id. Such a choice modifies the bottom coefficients of matrices introduced in Section 2.3, which become

$$
N=-\frac{1}{\operatorname{Re}}\left(\begin{array}{ll}
1 & 0 \\
0 & \frac{1}{2}
\end{array}\right) \text { and } P=-\frac{1}{\operatorname{Re}}\left(\begin{array}{cc}
0 & -\frac{i}{k} \lambda^{2} \\
\frac{i k}{2} & 0
\end{array}\right) .
$$

Consequently the expressions of matrices $\hat{H}_{i j}$ and of the spectral radius $\rho(\hat{H})$ are also modified, as well as the expression of corresponding transparent operators (which are no longer antisymmetric). However, in our opinion, there is no theoretical reason indicating that working with the symmetric form of the stress tensor should lead to a better convergence of the DD algorithm than working with the non symmetric form. This is confirmed by our results, since we checked this alternative symmetric approach in the simple case of approximation $\hat{S}^{-2,0}$, and obtained optimal spectral radii which differ by less than 0.01 from their corresponding "non symmetric" values. 


\subsection{Extension to more than two subdomains}

As seen previously, we restricted the present study to a decomposition into two subdomains. Increasing the number of subdomains is out of the scope this paper. However it is well known that the performances of previous (so called one-level) methods are not scalable when the number of subdomains becomes large, due to a lack of a global exchange of information. This can be fixed by an additional solve of a coarse problem, the size of which is of the order of the number of subdomains. Such a so called two-level approach was proposed first in [20], and has been further developed in many papers, including very recent ones (see e.g. $[4,25,28]$ and references herein).

\subsection{Synthesis}

This study leads to the conclusion that $S_{i}^{-1,0}$ operators are good candidates to provide efficient interface conditions for the Schwarz DDM algorithm applied to the Stokes problem (3). They allow for a rapid convergence, even without overlap, in all our test cases, and rely only on two free parameters. They compare favorably to the simple Dirichlet-Dirichlet, but also to the simpler approximation $S_{i}^{-2,0}$. Moreover, considering one or two additional parameters in approximations $S_{i}^{-1,1}$ and $S_{i}^{-1,2}$ is probably not worth given the additional difficulty in the optimization of these parameters and the relatively small improvement in the convergence rate. Note also that two-sided $S_{i}^{-2,0}$ operators are a good alternative.

This study was performed in the $2-\mathrm{D}(x, z)$ case. Extending it to the 3 -D case would be a direct extension of the present work, which would make the derivation of the approximations more complex, but would not require new methodological tools. Conversely, considering the time variable would represent a significant new step. Considering waveform relaxation DDM (also called global-in-time methods) would indeed require coming back to Navier-Stokes equations, dealing with the nonlinear advection term and including the time variable in all previous derivations. However it would allow for the use of different space and time steps in the different subdomains, which is an interesting practical feature.

Finally let us remind that the Schwarz formulation is a natural framework for coupling different models, which is an important motivation for the present work, in the prospect of coupling different models in fluid dynamics.

Acknowledgements This work was supported by the ANR through contract ANR-11-MONU005 (COMODO) and by the French national programme LEFE/INSU (project CoCoA).

\section{Appendix}

The optimal values of the spectral radius $\rho_{\text {opt }}$ and of the parameters $a_{i}$ and $b_{i}$ for the different test cases and for different sizes of the overlap $\delta$ are summarized in the following table. 


\begin{tabular}{|c|c|c|c|c|c|c|c|c|c|c|}
\hline & & \multicolumn{3}{|c|}{ Case 2} & \multicolumn{3}{|c|}{ Case 3} \\
\hline & & $\delta=0$ & $\Delta x$ & $2 \Delta x$ & $\delta=0$ & $\Delta x$ & $2 \Delta x$ & $\delta=0$ & $\Delta x$ & $2 \Delta x$ \\
\hline Dir-Dir & $\rho$ & 1. & .978 & .955 & 1. & .951 & .864 & 1. & .999 & .998 \\
\hline \multirow{3}{*}{$\begin{array}{l}\hat{S}_{i}^{-2,0} \\
\text { one sided }\end{array}$} & $\rho$ & 1. & .3059 & .2213 & 1. & .1419 & .0813 & 1. & .4616 & .3863 \\
\hline & $a_{0}$ & & 229.88 & 284.72 & & 8.825 & 10.626 & & 61.734 & 75.140 \\
\hline & $\rho$ & 1. & .191 & & 1. & .093 & & 1. & .283 & \\
\hline \multirow[t]{2}{*}{ two sided } & $a_{0}$ & & 507.5 & & & 15 & & & 15 & \\
\hline & $a_{0}^{\prime}$ & & 69 & & & 4 & & & 171 & \\
\hline \multirow{3}{*}{$\hat{S}_{i}^{-2,2}$} & $\rho$ & 1. & .3048 & .22048 & 1. & .1400 & .0807 & 1. & .4614 & .3861 \\
\hline & $a_{0}$ & & 230.6 & 285.6 & & 8.90 & 10.66 & & 61.78 & 75.18 \\
\hline & $a_{2}$ & & $3.610^{-4}$ & $9.610^{-4}$ & & $2.010^{-4}$ & $2.410^{-4}$ & & $410^{-6}$ & $810^{-6}$ \\
\hline \multirow[t]{3}{*}{$\hat{S}_{i}^{-1,0}$} & $\rho$ & .7327 & .0669 & .0380 & .3807 & .0400 & .0080 & .5954 & .3940 & .2648 \\
\hline & $a_{0}$ & 63.22 & 528.7 & 571.5 & 4.815 & 13.487 & 18.70 & 42.0 & 74.12 & 103.64 \\
\hline & $b_{0}$ & 0.064 & -0.50 & -0.5016 & 0.1794 & -0.1765 & -0.2305 & 0.985 & -0.11 & -0.382 \\
\hline \multirow{4}{*}{$\hat{s}_{i}^{-1,1}$} & $\rho$ & .6497 & .0660 & .0379 & .3320 & .0340 & .0078 & .5924 & .3556 & .2439 \\
\hline & $a_{0}$ & 85.45 & 505 & 571.5 & 5.0394 & 14.015 & 18.61 & 42.40 & 81.93 & 109.62 \\
\hline & $b_{0}$ & 4.59 & -0.50 & -0.5015 & 0.84 & -0.1652 & -0.231 & 0.944 & -0.0325 & -0.327 \\
\hline & $a_{1}$ & -0.0236 & $-910^{-4}$ & $-910^{-4}$ & -0.007 & 0.00846 & -0.001 & $-1.410^{-4}$ & 0.01025 & 0.01388 \\
\hline
\end{tabular}

\section{References}

1. Brakkee, E., Vuik, C., Wesseling, P.: Domain decomposition for the incompressible NavierStokes equations: solving subdomain problems accurately and inaccurately. Int. J. Num. Meth. Fluids 26, 1217-1237 (1998)

2. Bruneau, C.H., Saad, Y.: The 2D lid-driven cavity problem revisited. Comput. \& Fluids 35(3), 23-23 (2006)

3. Dolean, V., Nataf, F., Rapin, G.: Deriving a new domain decomposition method for the Stokes equations using the Smith factorization. Math. Comp. 78, 789-814 (2009)

4. Efendiev, Y., Galvis, J., Lazarov, R., Willems, J.: Robust domain decomposition preconditioners for abstract symmetric positive definite bilinear forms. ESAIM Math. Mod. Num. Anal. 46, 1175-1199 (2012)

5. Engquist, B., Majda, A.: Absorbing boundary conditions for the numerical simulation of waves. Math. Comp. 139, 629-651 (1977)

6. Erturk, E.: Discussions on driven cavity flow. Int. J. Num. Meth. Fluids 60, 275-294 (2009)

7. Gander, M.J.: Optimized Schwarz methods. SIAM Journal on Numerical Analysis (2006)

8. Gunzburger, M., Lee, H.: An optimization-based domain decomposition method for the Navier-Stokes equations. SIAM J. Num. Anal. 37, 1455-1480 (2000)

9. Halpern, L.: Artificial boundary conditions for incompletely parabolic perturbations of hyperbolic systems. SIAM J. Math. Anal. 22, 1256-1283 (1991)

10. Halpern, L., Schatzman, M.: Artificial boundary conditions for incompressible viscous flows. SIAM J. Math. Anal. 20, 308-353 (1989)

11. Hoang, T.: Space-time domain decomposition methods for mixed formulations of flow and transport problems in porous media. Ph.D. thesis, University of Pierre and Marie Curie (Paris VI) (2013)

12. Japhet, C., Nataf, F.: The best interface conditions for domain decomposition methods: absorbing boundary conditions. In: L. Tourrette, L. Halpern (eds.) Absorbing Boundaries and Layers, Domain Decomposition Methods. Applications to Large Scale Computations, pp. 348-373. Nova Science Publishers (2001)

13. Japhet, C., Nataf, F., Rogier, F.: The optimized order 2 method: application to convectiondiffusion problems. Future Generation Computer Systems 18(1), 17-30 (2001)

14. Kong, F., Ma, Y., Lu, J.: An optimization-based domain decomposition method for numerical simulation of the incompressible Navier-Stokes flows. Num. Meth. PDEs 27, 255-276 (2011)

15. Kumar, P.: Purely algebraic domain decomposition methods for the incompressible NavierStokes equations. ArXiv e-prints (2011)

16. Lions, P.L.: On the Schwarz alternating method I. In: R. Glowinski, G. Golub, G. Meurant, J. Périaux (eds.) Proceedings of the First International Conference on Domain Decomposition Methods, Domain Decomposition Methods for Partial Differential Equations, pp. 1-42. SIAM (1988)

17. Lions, P.L.: On the Schwarz alternating method III: a variant for nonoverlapping subdomains. In: T. Chan, R. Glowinski, J. Périaux, O. Widlund (eds.) Proceedings of the Third International Conference on Domain Decomposition Methods, Domain Decomposition Methods for Partial Differential Equations, pp. 202-223. SIAM (1990) 
18. Martin, V.: Schwarz waveform relaxation algorithms for the linear viscous equatorial shallow water equations. SIAM J. Sci. Comput. 31, 3595-3625 (2009)

19. Müller, L., Lube, G.: A nonoverlapping domain decomposition method for the nonstationary Navier-Stokes problem. ZAMM J. Appl. Math. Mech. 81, 725-726 (2001)

20. Nicolaides, R.: Deflation of conjugate gradients with applications to boundary value problems. SIAM J. Num. Anal. 24, 355-365 (1987)

21. Otto, F., Lube, G.: A nonoverlapping domain decomposition method for the Oseen equations. Math. Models Methods Appl. Sci. 8, 1091-1117 (1998)

22. Pavarino, L., Widlund, O.: Balancing Neumann-Neumann methods for incompressible Stokes equations. Comm. Pure Appl. Math. 55, 302-335 (2002)

23. Ronquist, E.: A domain decomposition solver for the steady Navier-Stokes equations. In: A. Ilin, L. Scott (eds.) Proceedings of ICOSAHOM-95, pp. 469-485. SIAM (1996)

24. Schwarz, H.A.: Über einen Grenzübergang durch alternierendes Verfahren. Vierteljahrsschrift der Naturforschenden Gesellschaft in Zürich 15, 272-286 (1870)

25. Spillane, N., Dolean, V., Hauret, P., Nataf, F., Pechstein, C., Scheichl, R.: Abstract robust coarse spaces for systems of pdes via generalized eigenproblems in the overlaps. Numer. Math. 126, 741-770 (2014)

26. Strikwerda, J., Scarbnick, C.: A domain decomposition method for incompressible flow. SIAM J. Sci. Comput. 14, 49-67 (1993)

27. Toselli, A., Widlund, O.: Domain decomposition methods - Algorithms and theory. Springer, Berlin-Heidelberg (2005)

28. Willems, J.: Robust multilevel methods for general symmetric positive definite operators. SIAM J. Num. Anal. 52, 103-124 (2014)

29. Xu, X., Chow, C., S.H., L.: On non overlapping domain decomposition methods for the incompressible Navier-Stokes equations. ESAIM Math. Mod. Num. Anal. 39, 1251-1269 (2005) 